\title{
HYPERELASTIC CURVES ALONG IMMERSIONS
}

\author{
BAYRAM ŞAHIN, GÖZDE ÖZKAN TÜKEL, AND TUNAHAN TURHAN
}

Received 15 December, 2020

\begin{abstract}
Hyperelastic curves in a Riemannian manifold are solutions of a constrained variational problem and characterized by Euler-Lagrange equations. We study the effect of hyperelastic curves on the geometry of isometric immersions. We investigate the relation between hyperelastic curves and umbilical submanifolds and apply the results to analyze classical elastic curves. The case of a Riemannian manifold with constant sectional curvature is also discussed and some applications are presented for illustrating the results.
\end{abstract}

2010 Mathematics Subject Classification: 53B20; 53A35

Keywords: hyperelastic curve, mean curvature vector field, Riemannian manifold, isometric immersion

\section{INTRODUCTION}

A parametrized curve by its arclength is called a hyperelastic curve if it is a critical point of the following curvature energy action defined on a suitable space of curves in a Riemannian manifold:

$$
\mathcal{F}_{\gamma}^{r}=\int\left(\kappa^{r}+\lambda\right) d s,
$$

where $\kappa$ denotes the curvature of $\gamma$ [3,21,22]. Such curves are called as free hyperelastic curves when $\lambda=0$. The functional $\mathcal{F}_{\gamma}^{r}$ is nothing but the classical EulerBernoulli's bending (or elastic) energy functional for $r=2$. Immersed curves which are critical for the bending energy functional satisfying some boundary conditions are called elastic curves (or elastica) [16]. The existence, classification or stability problems of elastic curves or their generalizations in Riemannian manifolds have attracted the attention of many researches. Some of the most remarkable examples in the literature worked by D. Singer et al. [13, 15, 16, 23]. J. Langer and D. Singer in 1984 showed that there exist closed elastic curves of a fixed length in a compact Riemannian manifold [16]. At around the same time with the paper [16], that idea is shown in [14] by different method [10]. These curves have been characterized on the sphere in Euclidean 3-space by Brunnett and Crouch [7]. Another noteworthy

This paper is supported by The Scientific and Technological Council of Turkey (TUBITAK)with number 119F025. 
works of elastic curves and its extensions have been studied by Garay et al. in nonEuclidean spaces $[1,3,10]$. They have found important results of this variational problem in Lorentz-Minkowski space.

The study of this topic in low dimensional cases, curves and surfaces, has important applications to Physics, Biophysics and Engineering while attention to higher dimensional submanifolds has usually shown a more purely mathematical flavor. However, the hyperelastic curves have important applications to other higher dimensional variational problems in submanifolds and physics such as Chen-Willmore submanifolds, string theory and $p$-branes, models of relativistic particles, capillary pressure difference, etc. $[3,10]$.

The special curves on the surfaces were first considered in Riemannian manifolds by Nomizu and Yano [18]. The authors described the concept of a circle and showed that if a circle on a submanifold is carried to the ambient manifold along an immersion, the submanifold is totally umbilical and the mean curvature vector field is parallel [18]. Ikawa expressed a characterization of a helix by a differential equation in a Riemannian manifold and obtained the necessary and sufficient condition as depending upon the mean curvature vector field that a helix in a Riemannian submanifold corresponds to a helix in ambient manifold [12,17, 18]. One of the most active area of differential geometry has been the theory of isometric immersions [9] and this theory is still active area, see for instance [2] and [4]. The aim of this paper is to consider hyperelastic curves along an immersion. By considering the role of submanifold theory in geometric mechanics [8] and applications of elastic curves, we believe our result will start a fresh research area by combining these two concepts.

In section 2, we give some geometric preliminaries concerning the basic problem of the paper mentioned in the introduction. In section 3, we examine isometric immersions which carry a hyperelastic curve of the total manifold to ambient manifold as a hyperelastic curve. Then, we give a characterization of the submanifold with regard to the mean curvature vector field $H$ by means of hyperelastic curves. We also consider elastic curves under isometric immersions. In the last section, we give some results according to constant sectional curvature of the manifold. Finally, we exemplify the findings.

\section{GEOMETRICAL NOTATIONS AND PRELIMINARIES}

Let $M$ be a $n$-dimensional submanifold of a $m$-dimensional Riemannian manifold $\bar{M}$. $\langle\cdot, \cdot\rangle$ denotes both the metric tensor fields of $\bar{M}$ and the induced metric of $M$. A vector field $V$ in $\bar{M}$ is called a normal vector field in $M$ if $\langle X, V\rangle=0$ for any vector field $X$ in $M$ and a unit normal vector field in $M$ is known a normal section, [24]. $\bar{\nabla}$ and $\nabla$ denote the Riemannian connection of $\bar{M}$ and induced connection of $M$ respectively. $\chi(\bar{M})$ and $\chi(M)$ are the set of the Lie algebra of vector fields in $\bar{M}$ and $M$, respectively. $\chi^{\perp}(M), T M$ and $T M^{\perp}$ will express the set of all normal vector fields of $M$, the tangent bundle and the normal vector bundle of $M$, respectively. 
Let $X$ and $Y$ be vector fields on $M$. The Gauss formula [24] is given by

$$
\bar{\nabla}_{X} Y=\nabla_{X} Y+h(X, Y),
$$

where $\nabla_{X} Y$ is the tangential component, $h(X, Y)$ is the normal component of $\bar{\nabla}_{X} Y$. $h(X, Y)$ is the second fundamental form of $M . h$ is a bilinear and symmetric form in $X$ and $Y$.

Let $V$ be a normal vector field in $M$. The Weingarten formula is given by

$$
\bar{\nabla}_{X} V=-A_{V} X+D_{X} V
$$

where $D$ is the connection in the normal bundle and $A$ is the shape operator of $M$. $A$ and $h$ satisfy

$$
\left\langle A_{V} X, Y\right\rangle=\langle h(X, Y), V\rangle .
$$

From the Gauss and Weingarten formulas, we have

$$
\bar{R}(X, Y) Z=R(X, Y) Z-A_{h(Y, Z)} X+A_{h(X, Z)} Y+\left(\bar{\nabla}_{X} h\right)(Y, Z)-\left(\bar{\nabla}_{Y} h\right)(X, Z),
$$

where $\bar{R}$ and $R$ are the Riemannian curvature tensor fields of $\bar{M}$ and $M$, respectively. In addition, $\left(\bar{\nabla}_{X} h\right)(Y, Z)$ and $\left(\nabla_{X} A\right)_{V} Y$ are defined as the covariant derivative of the second fundamental forms $h$ and $A$ as follows

$$
\left(\bar{\nabla}_{X} h\right)(Y, Z)=D_{X} h(Y, Z)-h\left(\nabla_{X} Y, Z\right)-h\left(Y, \nabla_{X} Z\right)
$$

and

$$
\left(\nabla_{X} A\right)_{V} Y=\nabla_{X}\left(A_{V} Y\right)-A_{D_{X} V} Y-A_{V} \nabla_{X} Y
$$

respectively. On the other hand, the following equality is satisfied

$$
\left\langle\left(\bar{\nabla}_{X} h\right)(Y, Z), V\right\rangle=\left\langle\nabla_{X}(A)_{V} Y, Z\right\rangle .
$$

$M$ is called a totally geodesic submanifold in $\bar{M}$ if its second fundamental form is identically zero, that is $h=0$ or equivalently $A=0$. If the second fundamental form satisfies

$$
h(X, Y)=\langle X, Y\rangle H,
$$

then $M$ is said to be totally umbilical. If the vector $h(x, x)$ has the same length for any unit vector $x \in T_{p} M$, then $M$ is called isotropic at $p$. A submanifold $M$ is isotropic iff $h$ satisfies

$$
\langle h(x, x), h(x, y)\rangle=0
$$

for any orthonormal vectors $x$ and $y$ of $T_{p} M$. Furthermore if

$$
h(x, y)=0
$$

for any orthonormal vectors $x$ and $y$ of $T_{p} M$, then $M$ is totally umbilical at $p[12,19]$. 


\section{Characterization OF The SUbMANifold OF A RiEMANNiAN MANifold BY HYPERELASTIC CURVES}

We firstly remind a characterization of the hyperelastic curve in a Riemannian manifold. We consider the family of $C^{\infty}$ curves as follows

$$
\mathfrak{I}=\left\{\gamma \mid \gamma:[0, \ell] \subset \mathbb{R} \rightarrow M, \gamma(i \ell)=p_{i}, \quad p_{i} \in M, \dot{\gamma}(i \ell)=v_{i}, \quad v_{i} \in T_{p_{i}} M i=0,1\right\}
$$

For a parametrized curve $\gamma \in \mathfrak{I}, \kappa$ is the geodesic curvature of $\gamma$. Then a hyperelastic curve is a critical point of the functional $\mathcal{F}_{\gamma}^{r}: \mathfrak{I} \rightarrow[0, \infty)$ defined by (1.1) for a natural number $r \geq 2$ (see [3]).

Let $\gamma: I \rightarrow M$ be an immersed unit speed curve in a $n$-dimensional Riemannian manifold $M$. We denote by $T, N$ and $B$, the unit tangent vector field, the unit normal vector field and the binormal vector field of $\gamma$, respectively. $\kappa=\left\|\nabla_{T} T\right\|$ is the geodesic curvature and $\tau=-\left\langle\nabla_{T} B, N\right\rangle$ is the torsion of $\gamma \cdot \gamma$ has also curvatures $\kappa_{1}=\kappa>0, \kappa_{2}=\tau, \kappa_{3}, \kappa_{4}, \ldots, \kappa_{n-1}$ and Frenet frame $N_{0}=T, N_{1}=N, N_{2}=B$, $N_{3}, N_{4}, \ldots, N_{n-1}$. Then, the Frenet equations are given by

$$
\nabla_{T} N_{i}=-\kappa_{i} N_{i-1}+\kappa_{i+1} N_{i+1}, \quad 0 \leq i \leq n-1
$$

(defining $\kappa_{0}=\kappa_{n}=0$ ) see [16]. In this case $\gamma$ is called a Frenet curve of order $\mathrm{n}$.

Critical points of the functional (1.1) are characterized by the Euler-Lagrange equation

$$
\nabla_{T}^{2}\left(\kappa^{r-2} \nabla_{T} T\right)+\kappa^{r-2} R\left(\nabla_{T} T, T\right) T+\nabla_{T}(\lambda T)=0
$$

for some constant $b \in \mathbb{R}$ and

see $[3,21]$.

$$
\lambda=\frac{2 r-1}{r} \kappa^{r}+b
$$

Lemma 1. Let $M$ and $\bar{M}$ be Riemannian manifolds and $i: M \rightarrow \bar{M}$ an isometric immersion such that $\gamma$ is a curve on $M$. Assume that $\bar{\gamma}(s)=i \circ \gamma(s)$ is a curve with curvature $\bar{\kappa}$ in $\bar{M}$. Then we have the following equations:

(i)

$$
\begin{aligned}
\bar{\nabla}_{T}^{2}\left(\bar{\kappa}^{r-2} \bar{\nabla}_{T} T\right)= & \nabla_{T}^{2}\left(\xi \nabla_{T} T\right)+h\left(T, \nabla_{T}\left(\xi \nabla_{T} T\right)\right)-A_{\xi h\left(T, \nabla_{T} T\right)} T \\
& +D_{T} \xi h\left(T, \nabla_{T} T\right)-\nabla_{T}\left(A_{\xi h(T, T)} T\right)-h\left(A_{\xi h(T, T)} T, T\right) \\
& -A_{D_{T} \xi h(T, T)} T+D_{T}^{2} \xi h(T, T),
\end{aligned}
$$

(ii)

$$
\begin{aligned}
\bar{\kappa}^{r-2} \bar{R}\left(\bar{\nabla}_{T} T, T\right) T= & \xi R\left(\nabla_{T} T, T\right) T-\xi A_{h(T, T)} \nabla_{T} T+\xi A_{h\left(\nabla_{T} T, T\right)} T \\
& +\xi\left(\bar{\nabla}_{\nabla_{T} T} h\right)(T, T)-\xi\left(\bar{\nabla}_{T} h\right)\left(\nabla_{T} T, T\right),
\end{aligned}
$$

where $\xi=\left(\kappa^{2}+\|h(T, T)\|^{2}\right)^{\frac{r-2}{2}}$ and $\kappa$ is the curvature of $\gamma$ in $M$. 
Proof. Suppose that $\bar{\gamma}(s)$ is an immersed curve with $\bar{\kappa}$ in $\bar{M}$.

(i) From Gauss and Weingarten formulas, we have

$$
\begin{aligned}
\bar{\nabla}_{T}\left(\bar{\kappa}^{r-2} \bar{\nabla}_{T} T\right) & =\bar{\nabla}_{T}\left(\xi \nabla_{T} T\right)+\bar{\nabla}_{T}(\xi h(T, T)) \\
& =\nabla_{T}\left(\xi \nabla_{T} T\right)+\xi h\left(T, \nabla_{T} T\right)-A_{\xi h(T, T)} T+D_{T} \xi h(T, T) .
\end{aligned}
$$

Taking derivative of (3.3) and using (2.1) and (2.2), we get the desired equation. (ii) From (2.1) and (2.4), we have

$$
\begin{aligned}
\bar{R}\left(\bar{\nabla}_{T} T, T\right) T= & R\left(\nabla_{T} T, T\right) T-A_{h(T, T)} \nabla_{T} T+A_{h\left(\nabla_{T} T, T\right)} T \\
& +\left(\bar{\nabla}_{\nabla_{T} T} h\right)(T, T)-\left(\bar{\nabla}_{T} h\right)\left(\nabla_{T} T, T\right) .
\end{aligned}
$$

Taking inner product both sides of (3.4) with $\xi$, we obtain the relation (ii) of Lemma (1).

Now, we suppose that $\bar{\gamma}(s)$ is a hyperelastic curve in $\bar{M}$. From (3.1), we have the following equation

$$
\bar{\nabla}_{T}^{2}\left(\bar{\kappa}^{r-2} \bar{\nabla}_{T} T\right)+\bar{\kappa}^{r-2} \bar{R}\left(\bar{\nabla}_{T} T, T\right) T+\bar{\nabla}_{T}(\lambda T)=0,
$$

for some constant $b \in \mathbb{R}$ and

$$
\lambda=\frac{2 r-1}{r} \overline{\mathrm{\kappa}}^{r}+b .
$$

Taking into consideration Lemma 1, we obtain

$$
\begin{array}{r}
\nabla_{T}^{2}\left(\xi \nabla_{T} T\right)+h\left(T, \nabla_{T}\left(\xi \nabla_{T} T\right)\right)+D_{T} \xi h\left(T, \nabla_{T} T\right)-\nabla_{T}\left(A_{\xi h(T, T)} T\right) \\
-\xi h\left(A_{h(T, T)} T, T\right)-A_{D_{T} \xi h(T, T)} T+D_{T}^{2} \xi h(T, T)+\xi R\left(\nabla_{T} T, T\right) T \\
-\xi A_{h(T, T)} \nabla_{T} T+\xi\left(\bar{\nabla}_{\nabla_{T} T} h\right)(T, T)-\xi\left(\bar{\nabla}_{T} h\right)\left(\nabla_{T} T, T\right) \\
+\nabla_{T}(\lambda T)+\lambda h(T, T)=0,
\end{array}
$$

where

The tangent part of (3.7) is

$$
\lambda=\frac{2 r-1}{r}\left(\kappa^{2}+\| h\left(T, T \|^{2}\right)^{\frac{r}{2}}+b\right.
$$

$$
\begin{aligned}
& \nabla_{T}^{2}\left(\xi \nabla_{T} T\right)-\nabla_{T}\left(A_{\xi h(T, T)} T\right)-A_{D_{T} \xi h(T, T)} T \\
& +\xi R\left(\nabla_{T} T, T\right) T-\xi_{h(T, T)} \nabla_{T} T+\nabla_{T}(\lambda T)=0 .
\end{aligned}
$$

We have from (2.6) and (2.5)

$$
\nabla_{T}\left(A_{\xi h(T, T)} T\right)=\nabla_{T}(A)_{\xi h(T, T)} T+A_{D_{T} \xi h(T, T)} T+A_{\xi h(T, T)} \nabla_{T} T
$$

and

$$
\xi\left(\bar{\nabla}_{T} h\right)(T, T)=\xi D_{T} h(T, T)-2 \xi h\left(\nabla_{T} T, T\right),
$$


respectively. Substituting (3.9) and (3.10) into (3.8), we have

$$
\begin{aligned}
& \nabla_{T}^{2}\left(\xi \nabla_{T} T\right)-\nabla_{T} A_{\xi h(T, T)} T-2 A_{\left(D_{T} \xi\right) h(T, T)} T-2 \xi A_{\left(\bar{\nabla}_{T} h\right)(T, T)} T \\
& -4 \xi A_{h\left(\nabla_{T} T, T\right)} T-2 \xi A_{h(T, T)} \nabla_{T} T+\xi R\left(\nabla_{T} T, T\right) T+\nabla_{T}(\lambda T)=0 .
\end{aligned}
$$

From Frenet equations of $\gamma$, (3.11) reduces to

$$
\begin{aligned}
& \left(\lambda_{s}-3 \xi \kappa \kappa_{s}-2 \kappa^{2} \xi_{s}\right) T+\left(\xi \kappa_{s s}-\kappa^{3} \xi+2 \kappa_{s} \xi_{s}+\xi_{s s} \kappa-\kappa \tau^{2} \xi+\lambda \kappa\right) N \\
& +\left(2 \kappa_{s} \tau \xi+\kappa \tau_{s} \xi+2 \kappa \tau \xi_{s}\right) B-4 \xi \kappa A_{h(N, T)} T-2 \xi \kappa A_{h(T, T)} N \\
= & 2 A_{\left(D_{T} \xi\right) h(T, T)} T+2 \xi A_{\left(\bar{\nabla}_{T} h\right)(T, T)} T+\nabla_{T} A_{\xi h(T, T)} T-\xi \kappa R(N, T) T .
\end{aligned}
$$

Taking inner product with $T$ to (3.12) and using (3.10), we calculate

$$
\begin{aligned}
& \lambda_{s}-3 \xi \kappa \kappa_{s}-2 \kappa^{2} \xi_{s}-6 \xi \kappa\langle h(T, N), h(T, T)\rangle= \\
& 2\left\langle A_{\left(D_{T} \xi h\right)(T, T)} T, T\right\rangle-4 \kappa \xi\left\langle A_{h(T, N)} T, T\right\rangle+\left\langle\nabla_{T} A_{\xi h(T, T)} T, T\right\rangle
\end{aligned}
$$

by using the fact $\langle N, T\rangle=\langle B, T\rangle=\langle R(N, T) T, T\rangle=0$, (2.3) and (3.10). By using (2.3), (2.7) and (3.10) we have

$$
\begin{aligned}
\lambda_{s}-3 \xi \kappa \kappa_{s}-2 \kappa^{2} \xi_{s} & =2\left\langle h(T, T), D_{T} \xi h(T, T)\right\rangle+\left\langle\xi h(T, T), D_{T} h(T, T)\right\rangle \\
& =2\left(D_{T} \xi\right)\|h(T, T)\|^{2}+\frac{3}{2} \xi D_{T}\|h(T, T)\|^{2} \\
& =\xi\left((r-2)\left(\kappa^{2}+\|h(T, T)\|^{2}\right)^{-1}\|h(T, T)\|^{2}+\frac{3}{2}\right) D_{T}\|h(T, T)\|^{2} .
\end{aligned}
$$

The normal part of (3.7) is

$$
\begin{aligned}
h\left(T, \nabla_{T}\left(\xi \nabla_{T} T\right)\right) & +D_{T} \xi h\left(T, \nabla_{T} T\right)-\xi h\left(A_{h(T, T)} T, T\right)+D_{T}^{2} \xi h(T, T) \\
& +\xi\left(\bar{\nabla}_{\nabla_{T} T} h\right)(T, T)-\xi\left(\bar{\nabla}_{T} h\right)\left(\nabla_{T} T, T\right)+\lambda h(T, T)=0 .
\end{aligned}
$$

We have from (2.5)

$$
\begin{aligned}
\xi D_{T}^{2} h(T, T)= & \xi\left(\bar{\nabla}_{T}^{2} h\right)(T, T)+4 \xi D_{T} h\left(T, \nabla_{T} T\right) \\
& -2 \xi h\left(\nabla_{T} T, \nabla_{T} T\right)-2 \xi h\left(T, \nabla_{T}^{2} T\right) .
\end{aligned}
$$

Substituting (3.15) into (3.14) and using (2.5), we get

$$
\begin{array}{r}
h\left(T, \xi_{s} \nabla_{T} T\right)+4 \xi h\left(T, \nabla_{T}^{2} T\right)+5\left(D_{T} \xi\right) h\left(T, \nabla_{T} T\right)+4 \xi\left(\bar{\nabla}_{T} h\right)\left(T, \nabla_{T} T\right) \\
+3 \xi h\left(\nabla_{T} T, \nabla_{T} T\right)-\xi h\left(A_{h(T, T)} T, T\right)+\left(D_{T}^{2} \xi\right) h(T, T)+2\left(D_{T} \xi\right)\left(\bar{\nabla}_{T} h\right)(T, T) \\
+\xi\left(\bar{\nabla}_{T}^{2} h\right)(T, T)+\xi\left(\bar{\nabla}_{\nabla_{T} T} h\right)(T, T)+\lambda h(T, T)=0 .
\end{array}
$$


Taking into consideration the Frenet equations, we have

$$
\begin{array}{r}
\left(\kappa \xi_{s}+4 \xi \kappa_{s}+5\left(D_{T} \xi\right) \kappa\right) h(T, N)+4 \xi \kappa \tau h(T, B)+4 \xi \kappa\left(\bar{\nabla}_{T} h\right)(T, N) \\
+3 \xi \kappa^{2} h(N, N)+2\left(D_{T} \xi\right)\left(\bar{\nabla}_{T} h\right)(T, T)+\xi \kappa\left(\bar{\nabla}_{N} h\right)(T, T)= \\
\left(4 \xi \kappa^{2}-D_{T}^{2} \xi\right) h(T, T)+\xi h\left(A_{h(T, T)} T, T\right)+\lambda h(T, T)-\xi\left(\bar{\nabla}_{T}^{2} h\right)(T, T) .
\end{array}
$$

Changing $B$ into $-B$ in (3.16) and using (3.16), we get

$$
h(T, B)=0 .
$$

In the following proposition, we give a characterization of the submanifold of a Riemannian manifold by using hyperelastic curves.

Proposition 1. Let $M$ and $\bar{M}$ be Riemannian manifolds and $i: M \rightarrow \bar{M}$ an isometric immersion such that $\gamma$ is a curve on $M$. Assume that $\bar{\gamma}(s)=i \circ \gamma(s)$ is a hyperelastic curve with curvature $\bar{\kappa}$ in $\bar{M}$. Then $M$ is an isotropic submanifold if one of the following conditions are satisfied.:

(i) $r=2$ that is $\bar{\gamma}(s)$ is an elastic curve,

(ii) curvature $\kappa$ of $\gamma$ is a constant when $\bar{\kappa} \neq 0$.

Proof. If $\bar{\gamma}=\bar{\gamma}(s)$ is a hyperelastic curve with curvature $\bar{\kappa}$ and $\gamma(s)$ has the curvature $\kappa$. Then we have the following cases:

(i) If $r=2$ then (3.13) reduces to

$$
D_{T}\|h(T, T)\|^{2}=0 .
$$

Thus $\|h(T, T)\|$ is equal to a constant value, that is, $M$ is an isotropic submanifold.

(ii) We suppose that $\kappa$ is a constant value. From (3.13), we obtain

$$
\xi\left((r-2)\left(\kappa^{2}+\|h(T, T)\|^{2}\right)^{-1}\|h(T, T)\|^{2}+\frac{3}{2}\right) D_{T}\|h(T, T)\|^{2}=0 .
$$

On the other hand, since $\bar{\kappa} \neq 0$ and $r \geq 2$, then we have (3.18).

From (2.8), (3.17) and Proposition 1, the proof of the following corollary is clear.

Corollary 1. We suppose that $\bar{\gamma}(s)=i \circ \gamma(s)$ is a hyperelastic curve with curvature $\bar{\kappa}$ in $\bar{M}$. In this case, $M$ is totally umbilic submanifold if it is isotropic.

Now, we introduce the concept of hyperelastic immersion.

Definition 1. Let $i$ be an isometric immersion from a Riemannian manifold $M$ to a Riemannian manifold $\bar{M}$ such that $\gamma$ is a hyperelastic curve on $M$. If the curve $i \circ \gamma$ is also a hyperelastic curve on $\bar{M}$, then the immersion $i$ is called a hyperelastic immersion. 
From (3.1) and the Frenet equations for $\gamma$, we have the following proposition (similar to that of [5]).

Proposition 2. Let $\gamma$ be a regular curve in $\mathbb{R}^{n}$ with curvatures $\{\kappa, \tau, \delta, \ldots\}$. Then $\gamma$ be a critical for the bending energy functional (1.1) if and only if the following Euler-Lagrange equations are satisfied

$$
\begin{aligned}
(r-1) \kappa_{s s}+(r-1)(r-2) \kappa^{-1} \kappa_{s}^{2}-\kappa \tau^{2}+\frac{r-1}{r} \kappa^{3} & =0 \\
2(r-1) \kappa_{s} \tau+\kappa \tau_{s} & =0 \\
\delta & =0 .
\end{aligned}
$$

Especially, a hyperelastic curve in $\mathbb{R}^{2}$ or $\mathbb{R}^{3}$ can be moved to a hyperelastic curve in $\mathbb{R}^{n}$ by a hyperelastic immersion. In the following, an example of a hyperelastic immersion is given.

Example 1. The mapping $j$ is defined as

$$
\begin{aligned}
j:[-1,1] \times I \subset \mathbb{R}^{2} & \rightarrow \mathbb{R}^{3} \\
(u, v) & \rightarrow\left(u, v, \sqrt{\frac{r-1}{r}} \arccos (u)\right) .
\end{aligned}
$$

Let

$$
\beta(s)=\left(\cos \left(\frac{s}{\sqrt{\frac{2 r-1}{r}}}\right), \sin \left(\frac{s}{\sqrt{\frac{2 r-1}{r}}}\right)\right) \text { where } 0 \leq\left(\frac{s}{\sqrt{\frac{2 r-1}{r}}}\right) \leq 2 \pi,
$$

be a curve in $\mathbb{R}^{2}$. Then we find the curve

$$
\bar{\beta}(s)=\left(\cos \left(\frac{s}{\sqrt{\frac{2 r-1}{r}}}\right), \sin \left(\frac{s}{\sqrt{\frac{2 r-1}{r}}}\right), \sqrt{\frac{r-1}{r}} \frac{s}{\sqrt{\frac{2 r-1}{r}}}\right) .
$$

We can see from (3.19) that $\beta$ and $\bar{\beta}$ are hyperelastic curves in $\mathbb{R}^{2}$ and $\mathbb{R}^{3}$, respectively. This implies that $j$ is a hyperelastic immersion.

The following theorem gives a result of hyperelastic immersions in terms of the elements of submanifold theory.

Theorem 1. Let $i$ be a hyperelastic immersion between the submanifold $M$ and $a$ Riemannian manifold $\bar{M}$. If $\gamma$ is a hyperelastic curve with the constant curvature $\mathrm{\kappa}$ and the tangent vector field $T$ in $M$, then $M$ is a totally umbilical submanifold and the mean curvature vector field $H$ satisfies

$$
D_{T}^{2} H=C H,
$$

where $C=\kappa^{2}+\|H\|^{2}+\frac{\lambda}{\xi}=$ constant. Conversely if the isometric immersion is totally geodesic, then it is a hyperelastic immersion. 
Proof. Let $i$ be a hyperelastic immersion between Riemannian manifolds $M$ and $\bar{M}$. Suppose that $\gamma(s)$ is a hyperelastic curve with a constant curvature $\kappa$ and the tangent vector field $T$ in $M$. Then $\gamma$ satisfies the Euler-Lagrange equation (3.1) with (3.2). We can see from Proposition 1, $M$ is an isotropic submanifold. Since $\bar{\gamma}$ is a hyperelastic curve in $\bar{M}$, we have (3.5) with (3.6). On the other hand Corollary 1 shows that $M$ is totally umbilical submanifold.

So, (3.16) reduces to

$$
\begin{aligned}
& 4 \xi \kappa\left(\bar{\nabla}_{T} h\right)(T, N)+3 \xi \kappa^{2} h(N, N)+\xi \kappa\left(\bar{\nabla}_{N} h\right)(T, T)= \\
& 4 \xi \kappa^{2} h(T, T)+\xi h\left(A_{h(T, T)} T, T\right)+\lambda h(T, T)-\xi\left(\bar{\nabla}_{T}^{2} h\right)(T, T) .
\end{aligned}
$$

Changing $N$ into $-N$ in (3.21), we have

$$
4 \xi \kappa\left(\bar{\nabla}_{T} h\right)(T, N)+\xi \kappa\left(\bar{\nabla}_{N} h\right)(T, T)=0 .
$$

Substituting (3.22) into (3.21) we have

$$
-\xi \kappa^{2} H=\xi\left\langle A_{h(T, T)} T, T\right\rangle H+\lambda H-\xi\left(\bar{\nabla}_{T}^{2} h\right)(T, T) .
$$

By using (2.3) and (3.15) we get

$$
-\xi \kappa^{2} H=\xi\|H\|^{2} H+\lambda H-\xi D_{T}^{2} H .
$$

Then, we obtain

$$
D_{T}^{2} H=\left(\kappa^{2}+\|H\|^{2}+\frac{\lambda}{\xi}\right) H .
$$

Conversely, we assume that the isotropic immersion is a totally geodesic. Since $\gamma$ is a hypereastic curve with the curvature $\kappa, i \circ \gamma$ satisfies the Euler-Lagrange equation (3.5) with (3.6).

Then we have the following result.

Corollary 2. If $i: M \rightarrow \bar{M}$ is a hyperelastic immersion and $\gamma$ is a hyperelastic curve with the constant curvature in $M$, then $\gamma$ has constant torsion.

Proof. Let $i$ be a hyperelastic immersion and $\gamma$ a hyperelastic curve with the constant curvature $\kappa$ in $M$. (3.12) can be written as

$$
\left(-\kappa^{2}-\tau^{2}+\frac{\lambda}{\xi}\right) N+\tau_{s} B=4 A_{h(N, T)} T+2 A_{h(T, T)} N .
$$

Taking inner product with $N$ in (3.23), we get

$$
\|H\|^{2}=\frac{1}{2}\left(-\kappa^{2}-\tau^{2}+\frac{\lambda}{\xi}\right) .
$$

Because of $\|h(T, T)\|=$ constant $=\|H\|$, one can see from (3.24), the torsion $\tau$ of $\gamma$ is a constant, too. 
We recall the following result which will be very crucial for our theorem. A unit speed curve $\gamma$ in a Riemannian manifold $M$ is called an elastic curve (or elastica) if it satisfies (3.1) with (3.2) for $r=2$ see [16,22]. The following theorem gives a characterization of totally umbilical submanifolds by the behaviour of elastic curves under an immersion.

Theorem 2. Let $i: M \rightarrow \bar{M}$ be an immersion from a Riemannian manifold $M$ to a Riemannian manifold $\bar{M}$ transporting each elastic curve with the tangent vector $T$, curvature $\kappa$ and torsion $\tau$, respectively, to another elastic curve on $\bar{M}$. Then $M$ is totally umbilical and the mean curvature vector field $H$ satisfies

$$
D_{T}^{2} H=\frac{1}{2}\left(\kappa^{2}-\tau^{2}+3 \lambda+\frac{\kappa_{s s}}{\kappa}\right) H, \quad\|H\|=\text { constant } .
$$

Conversely, if $M$ is an totally umbilical, its mean curvature vector satisfies (3.25) and $D_{\nabla_{T} T} H=2 \lambda H$ then an elastic curve in $M$ is an elastic curve in $\bar{M}$.

Proof. Suppose that $\gamma(s)$ is an elastic curve in $M$, with unit tangent vector field $T$ then the following equation is satisfied

$$
\left(\nabla_{T}\right)^{3} T+R\left(\nabla_{T} T, T\right) T+\nabla_{T}(\lambda T)=0
$$

with (3.2) for $r=2$. Since $\bar{\gamma}$ is an elastic curve with curvature $\bar{\kappa}$ in $\bar{M}$, we have also (3.5) with (3.6) for $r=2$. Proposition 1 and Corollary 1 show that $M$ is totally umbilical submanifold. If (3.23) is rewritten for $r=2$, and taking inner product with $N$, we get

$$
\|H\|^{2}=\frac{1}{2}\left(\frac{\kappa_{s s}}{\kappa}-\tau^{2}+\lambda-\kappa^{2}\right) .
$$

Now we consider for the similar process for the normal part in case of $r=2$. If necessary calculations are taken, we obtain

$$
D_{T}^{2} H=\left(\lambda+\kappa^{2}+\|H\|^{2}\right) H .
$$

Combining (3.26) and (3.27), we find (3.25).

Conversely, we assume that $M$ is totally umbilical and $H$ satisfies (3.25). On the other hand, we have

$$
\bar{\nabla}_{T}^{3} T=\nabla_{T}^{3} T-\nabla_{T}\left(\|H\|^{2} T\right)-\left(\kappa^{2}+\|H\|^{2}\right) H+D_{T}^{2} H .
$$

If we add $\bar{\kappa}^{r-2} \bar{R}\left(\bar{\nabla}_{T} T, T\right) T+\bar{\nabla}_{T}(\lambda T)$ both sides of (3.28), then we have from (2.1), (3.4) and (3.20)

$$
\begin{aligned}
\bar{\nabla}_{T}^{3}(T)+\bar{R}\left(\bar{\nabla}_{T} T, T\right) T+\bar{\nabla}_{T}(\lambda T)= & \nabla_{T}^{3}(T)+\nabla_{T}\left(\left(\lambda-2\|H\|^{2}\right) T\right) \\
& +R\left(\nabla_{T} T, T\right) T+2 \lambda H-\left(D_{\nabla_{T} T}\right) H .
\end{aligned}
$$

Since $\gamma$ is an elastic curve in $M$, the tangent part of (3.29) is calculated as

$$
\left(\nabla_{T}\right)^{3} T+\nabla_{T}(\lambda T)+R\left(\nabla_{T} T, T\right) T=0,
$$


where

$$
\lambda=\frac{3}{2} \kappa^{2}+\bar{b}
$$

for suitable choosing $\bar{b}=b+2\|H\|^{2}$. Normal part of (3.29) is found zero if

$$
D_{\nabla_{T} T} H=2 \lambda H .
$$

\section{HYPERELASTIC IMMERSIONS IN CONSTANT SECTIONAL CURVATURE MANIFOLDS}

Let $M$ be a Riemannian manifold having constant sectional curvature $c$. Then we have

$$
R(X, Y) Z=c(\langle Y, Z\rangle X-\langle X, Z\rangle Y)
$$

From (3.1) the following result is concluded: a unit speed curve $\gamma=\gamma(s)$ parametrized by arc length $s$ is a hyperelastic curve if it satisfies

$$
\nabla_{T}^{2}\left(\kappa^{r-2} \nabla_{T} T\right)+c \kappa^{r-2} \nabla_{T} T+\nabla_{T}(\lambda T)=0
$$

with (3.2) (see $[11,21])$

We know that a totally umbilical submanifold $M$ of a Riemannian manifold $\bar{M}$ of constant sectional curvature $\bar{c}$ has also constant sectional curvature $\bar{c}+\|H\|^{2}$ for $\operatorname{dim}(M)>2$ (see [24]). Thus, we can get the following corollary as a result of Theorem 1 and Corollary 1.

Corollary 3. Let $i$ be a hyperelastic immersion between the submanifold $M$ $(\operatorname{dim}(M)>2)$ and a Riemannian manifold $\bar{M}$ with constant sectional curvature $\bar{c}$. If $M$ is an isotropic submanifold of $\bar{M}$, then $M$ has also constant sectional curvature $c=\bar{c}+\|H\|^{2}$, where $H$ is the mean curvature vector field.

We can give the following corollary in a Riemannian manifold with constant sectional curvature as a result of Theorem 2 .

Corollary 4. Let $i: M \rightarrow \bar{M}(c)$ be a hyperelastic immersion between a Riemannian manifold $M$ and a Riemannian manifold $\bar{M}(c)$ of constant curvature such that $\gamma$ is an elastic curve with curvature $\kappa$, the torsion $\tau$ and the unit vector field $T$. Then $M$ is totally umbilical and the mean curvature vector field $H$ satisfies

$$
D_{T}^{2} H=\left(\frac{\kappa_{s s}}{\kappa}-\tau^{2}\right) H, \quad\|H\|=\text { constant } .
$$

Conversely, if $M$ is totally umbilical submanifold of a Riemannian manifold $\bar{M}$ with constant sectional curvature $c$ and the mean curvature vector field satisfies (4.2), then an elastic curve $\gamma$ is an elastic curve in $\bar{M}$. 
A Riemannian manifold of constant sectional curvature is called elliptic, hyperbolic or locally Euclidean if the sectional curvature is respectively positive, negative or zero. Now, we give an example of elastic immersion which carry elastic curves of the total manifold with 2-dimensional ( $c=0$, Euclidean plane) to ambient manifold with 3-dimensional ( $c=1,2$-sphere). Firstly, we recall some results for elastic curves on the plane and a 2-sphere $S^{2}$. An elastic curve on Euclidean plane satisfies the following differential equation

$$
\kappa^{\prime \prime}+\frac{1}{2} \kappa^{3}-\frac{1}{2} \lambda \kappa=0
$$

see [6]. On the other hand, Brunnett and Crouch classify the forms of spherical elastic curve based on the differential equation

$$
2 \kappa_{g}^{\prime \prime}+\kappa_{g}^{3}+\left(\frac{2}{\rho^{2}}-\sigma\right) \cdot \kappa_{g}=0
$$

where $\kappa_{g}$ is the geodesic curvature, $\rho$ is the tension parameter and $\sigma$ is the radius of the sphere, [7].

Example 2. Let $u$ and $v$ be coordinate functions of $\mathbb{R}^{2}$. The mapping $i$ is defined as

$$
\begin{aligned}
& i: \mathbb{R}^{2} \rightarrow S^{2} \subset \mathbb{R}^{3} \\
& (u, v) \rightarrow\left(u^{2}-v^{2}, 2 u v, 0\right)
\end{aligned}
$$

(see [20] ), where $\left(u^{2}+v^{2}\right)^{2}=1$. Let $\alpha: I \subset \mathbb{R} \rightarrow \mathbb{R}^{2}$ be a curve such that $t \rightarrow \alpha(t)=$ $(\cos t, \sin t)$, where $0 \leq t \leq 2 \pi$. We see from (4.3) that $\alpha$ is an elastic curve since the curvature $\kappa$ of $\alpha$ is 1 . The curve $i \circ \alpha$ is obtained as $(i \circ \alpha)(t)=(\cos 2 t, \sin 2 t, 0)$. One can see from (4.4) this curve is an elastica on $S^{2}$. Then $i$ is an elastic immersion.

\section{REFERENCES}

[1] "Ikergeometryteam," www.ikergeometry.org, date of Access: 25.05.2019.

[2] M. Aquib, J. Lee, J. Vilcu, and D. W. Yoon, "Classification of casorati ideal lagrangian submanifolds in complex space forms." Differential Geometry and its Applications, vol. 63, pp. 30-49, 2019, doi: 10.1016/j.difgeo.2018.12.006.

[3] J. Arroyo, O. Garay, and M. Mencia, "Closed free hyperelastic curves in real space forms." Proceeding of the XII Fall Workshop on Geometry and Physics, pp. 1-13, 2002.

[4] M. Aydin and I. Mihai, "Wintgen inequality for statistical surfaces." Math. Inequal. Appl., vol. 22, no. 1, pp. 123-132, 2019, doi: 10.7153/mia-2019-22-09.

[5] M. Barros, A. F. P. Lucas, and M. Merono, "Willmore tori and Willmore-Chen submanifolds in pseudo-Riemannian spaces." Journal of Geometry and Physics, vol. 28, pp. 45-46, 1998, doi: 10.1016/S0393-0440(98)00010-2.

[6] G. Brunnett, "A new characterization of plane elastic." Mathematical Methods in Computer Aided Design II., pp. 43-56, 1992, doi: 10.1016/B978-0-12-460510-7.50009-4.

[7] G. Brunnett and P. Crouch, "Elastic curves on the sphere." Adv. Comput. Math., vol. 2, no. 1, pp. 23-40, 1994, doi: 10.1007/BF02519034.

[8] F. Bullo and A. Lewis, Geometric Control of Mechanical Systems. New York: Springer, 2005. doi: 10.1007/978-1-4899-7276-7. 
[9] B. Chen, Total mean curvature and submanifolds of finite type. USA: World Scientific, 1984. doi: $10.1142 / 9237$.

[10] O. Garay, "Riemannian submanifolds shaped by the bending energy and its allies." Proceedings of The Sixteenth International Workshop on Diff. Geometry, vol. 16, pp. 57-70, 2021.

[11] R. Huang, "A note on the p-elastica in a constant curvature manifold." Journal of Geometry and Physics, vol. 49, pp. 343-349, 2004, doi: 10.1016/S0393-0440(03)00107-4.

[12] T. Ikawa, "On some curves in Riemannian geometry." Sooc. Jour. of Math., vol. 7, pp. 37-44, 1981.

[13] T. Ivey and D. Singer, "Knot types, homotopies and stability of closed elastic rods." Proceedings of the London Mathematical Society, vol. 79, no. 2, pp. 429-450, 1999, doi: 10.1112/S0024611599011983.

[14] N. Kosio, "Elasticae in a Riemannian submanifold." Osaka J. Math., vol. 29, no. 3, pp. 539-543, 1992.

[15] J. Langer and D. Singer, "Knotted elastic curves in $\mathbb{R}^{3}$." Journal of the London Mathematical Society, vol. 2, no. 3, pp. 512-520, 1984, doi: 10.1112/jlms/s2-30.3.512.

[16] J. Langer and D. Singer, "The total squared curvature of closed curves." Journal of Differential Geometry, vol. 20, pp. 1-22, 1984, doi: 10.4310/jdg/1214438990.

[17] S. Maeda, "A characterization of constant isotropic immersions by circles." Archiv der Mathematik, vol. 81, no. 1, pp. 90-95, 2003, doi: 10.1007/s00013-003-4677-1.

[18] K. Nomizu and K. Yano, "On circles and spheres in Riemannian geometry." Mathematische Annalen, vol. 210, no. 2, pp. 163-170, 1974, doi: 10.1007/BF01360038.

[19] B. O'Neill, "Isotropic and Kähler immersions." Canadian Journal of Mathematics, vol. 17, no. 6, pp. 907-915, 1965, doi: 10.4153/CJM-1965-086-7.

[20] B. O’Neill, Elementary differential geometry. New York: Academic Press, $1997 . \quad$ doi: 10.1016/C2009-0-05241-6.

[21] G. Ozkan Tukel, T. Turhan, and A. Yucesan, "Hyperelastic Lie quadratics." Honam Mathematical Journal, vol. 41, no. 2, pp. 369-380, 2019, doi: 10.5831/HMJ.2019.41.2.369.

[22] T. Popiel and L. Noakes, "Elastica in SO(3)." J. Aust. Math. Soc., vol. 83, pp. 105-124, 2007, doi: $10.1017 /$ S 1446788700036417.

[23] D. Singer, Lectures on elastic curves and rods. New York: In AIP Conference Proceedings American Institute of Physics, 2008. doi: 10.1063/1.2918095.

[24] K. Yano and M. Kon, Structures on manifolds. New York: World Scientific, $1984 . \quad$ doi: 10.1142/0067.

Authors' addresses

Bayram Şahin

Ege University, Science Faculty, Department of Mathematics, İzmir, Turkey

E-mail address: bayram. sahineege.edu.tr

Gözde Özkan Tükel

Isparta University of Applied Sciences, Isparta, Turkey

E-mail address: gozdetukeleisparta.edu.tr

Tunahan Turhan

(Corresponding author) Süleyman Demirel University, Isparta, Turkey

E-mail address: tunahanturhanesdu.edu.tr 